\title{
Effect of Dissolved Oxygen Tension on the Metabolism of Methylated Amines in Hyphomicrobium X in the Absence and Presence of Nitrate: Evidence for 'Aerobic' Denitrification
}

\author{
By J. B. M. MEIBERG, P. M. BRUINENBERG AND W. HARDER* \\ Laboratory of Microbiology, Biological Centre, University of Groningen, Kerklaan 30, \\ 9751 NN Haren, The Netherlands.
}

(Received 11 March 1980)

\begin{abstract}
Hyphomicrobium $\mathrm{X}$ possesses two different enzymes for the oxidation of dimethylamine, namely dimethylamine dehydrogenase and dimethylamine mono-oxygenase. During growth of the organism in batch culture at dissolved oxygen tensions (DOT) in excess of $30 \mathrm{mmHg}$ in media containing trimethylamine as the carbon and energy source, dimethylamine mono-oxygenase was the only enzyme involved in dimethylamine oxidation. The apparent $K_{\mathrm{m}}$ of the mono-oxygenase for oxygen was relatively high $(23.2 \mu \mathrm{M})$. The enzyme was less sensitive to inhibition by trimethylamine $\left(K_{1} 4.2 \mathrm{mM}\right)$ than was dimethylamine dehydrogenase $\left(K_{1} 7 \cdot 1 \mu \mathrm{M}\right)$ and therefore dimethylamine did not accumulate in the culture medium under these conditions. This was in contrast to observations made during anaerobic growth on trimethylamine. During growth of the organism in dimethylamine-limited chemostat cultures, the specific activities of the mono-oxygenase and the dehydrogenase were dependent on the DOT in the culture. When the DOT in the culture growing at a dilution rate of $0 \cdot 10 \mathrm{~h}^{-1}$ was decreased below $30 \mathrm{mmHg}$, the activity of the mono-oxygenase also decreased. In contrast, the activity of dimethylamine dehydrogenase increased, indicating that this enzyme gradually took over at the lower DOT. Below values of $5 \mathrm{mmHg}$ the culture became oxygen-limited and below $3 \mathrm{mmHg}$ it was washed out. When the organism was grown at low DOT in medium supplemented with nitrate, essentially the same results were obtained, except that wash-out of the culture under anaerobic conditions did not occur. The organism was able to carry out denitrification under 'partly aerobic' conditions (DOT 0 to $20 \mathrm{mmHg}$ at a dilution rate of $0 \cdot 10 \mathrm{~h}^{-1}$ ). Over this range of DOT, the monooxygenase hardly played a role in dimethylamine oxidation in vivo because the enzyme was inhibited by the nitrite which accumulated in the culture to a concentration of $3 \mathrm{~mm}$. The potential of Hyphomicrobium X to denitrify in the presence of oxygen was dependent on the growth rate of the organism. At low growth rates $\left(0.01 \mathrm{~h}^{-1}\right)$ synthesis of nitrate reductase, which largely determined the rate of nitrate reduction in the culture, was already significant at relatively high DOT (up to $50 \mathrm{mmHg}$ ). At high growth rates $\left(0.15 \mathrm{~h}^{-1}\right)$ nitrate reductase activity became apparent only at DOT values below $6 \mathrm{mmHg}$. The kinetics and the possible ecological significance of this 'aerobic' denitrification process are discussed.
\end{abstract}

\section{INTRODUCTION}

Hyphomicrobium $\mathrm{X}$ is able to utilize methylated amines under both aerobic and anaerobic conditions. A pathway for the metabolism of these carbon compounds in Hyphomicrobium $\mathrm{X}$ has been proposed (Meiberg \& Harder, 1978). It was shown that trimethylamine was oxidized by trimethylamine dehydrogenase, and dimethylamine, which is the product of 
trimethylamine oxidation, by dimethylamine dehydrogenase. Some properties of the latter enzyme have been described (Meiberg \& Harder, 1979).

A number of bacteria capable of aerobic growth on methylated amines have been shown to oxidize dimethylamine by way of a dimethylamine mono-oxygenase (Eady et al., 1971; Hampton \& Zatman, 1973; Colby \& Zatman, 1973, 1975; Loginova \& Trotsenko, 1976). This enzyme has also been detected in some hyphomicrobia when grown under aerobic conditions (Eady et al., 1971; Loginova et al., 1976). Although in the past dimethylamine dehydrogenase activity had been detected in aerobically grown Hyphomicrobium X (Meiberg \& Harder, 1978), in later experiments these results could not be repeated. Instead, monooxygenase activity was now detected, while the dehydrogenase was the only enzyme found to oxidize dimethylamine in anaerobically grown cells. During growth at low dissolved oxygen tensions (DOT 2 to $20 \mathrm{mmHg} ; 1 \mathrm{mmHg} \approx 133 \mathrm{~Pa}$ ) both the dehydrogenase and monooxygenase were present. It thus appears that Hyphomicrobium $\mathrm{X}$ has two enzymes available for the oxidation of dimethylamine; the relative contribution of each of these enzymes to dimethylamine oxidation is most probably governed by oxygen.

It is evident that the DOT may influence the activity of dimethylamine mono-oxygenase directly because oxygen is a substrate for this enzyme. Apart from an effect on its activity, oxygen may also directly or indirectly affect the synthesis of the mono-oxygenase. In addition, the synthesis and activity of other catabolic and respiratory enzymes may be influenced by the DOT. A limited supply of oxygen has the general effect that the growth rate of the organism is decreased until, when the DOT is zero, growth is no longer possible. However, since hyphomicrobia are able to denitrify, they can overcome this decrease in growth rate by anaerobic respiration when nitrate is present in the medium as a terminal electron acceptor instead of or in addition to oxygen. Indeed, Hirsch \& Conti (1964) and T. G. Wilkinson \& G. Hamer (unpublished) found that hyphomicrobia carry out denitrification at low DOT. It was therefore decided to study the metabolism of methylated amines by Hyphomicrobium $\mathrm{X}$ grown in the presence and absence of nitrate at various dissolved oxygen concentrations and at different specific growth rates.

\section{METHODS}

Maintenance, growth, preparation of cell-free extracts and chemical estimations. These were as described previously (Meiberg \& Harder, 1978, 1979).

Batch culture experiments. Aerobic growth of Hyphomicrobium $\mathrm{X}$ in mineral salts medium, supplied with methylated amines as growth substrates, was performed in a pH- and temperature-controlled batch fermenter as described previously (Meiberg \& Harder, 1978).

Continuous culture experiments. The organism was grown in chemostat cultures with dimethylamine or trimethylamine as the carbon and energy source, using a $1 \mathrm{l}$ (working volume) fermenter of the type described by Harder et al. (1974). The medium contained (per litre): $\left(\mathrm{NH}_{4}\right)_{2} \mathrm{SO}_{4}, 1.0 \mathrm{~g} ; \mathrm{MgSO}_{4} .7 \mathrm{H}_{2} \mathrm{O}, 0.2 \mathrm{~g} ; \mathrm{KH}_{2} \mathrm{PO}_{4}$ $1.0 \mathrm{~g}$; and trace element solution (Vishniac \& Santer, 1957), $0.2 \mathrm{ml}$. Where indicated, $\mathrm{KNO}_{3}$ was added to the medium to a final concentration of $20 \mathrm{~mm}$. When dimethylamine was the growth substrate, it was added to the medium, prior to heat-sterilization, to a final concentration of $15 \mathrm{~mm}$. Trimethylamine was filtersterilized before addition to the sterile mineral medium to a final concentration of $10 \mathrm{~mm}$. During growth, the pH was maintained at 7.0 by automatic addition of sterile $1 \mathrm{M}-\mathrm{NaOH}$ or $1 \mathrm{M}-\mathrm{H}_{2} \mathrm{SO}_{4}$, depending on the DOT. The temperature was maintained at $30^{\circ} \mathrm{C}$. The DOT in the culture was recorded with a steam-sterilizable Mackereth-type oxygen-electrode which was calibrated in sterile medium $\left(\right.$ at $\left.30^{\circ} \mathrm{C}\right)$. Air-saturated medium was assumed to have a DOT of $159 \mathrm{mmHg}$. After the DOT had been set at the desired value in a growing culture, it was maintained at this value $( \pm 0.5 \mathrm{mmHg})$ by automatic adjustment of the stirrer speed. Under these conditions it was determined that a dilution rate of $0.05 \mathrm{~h}^{-1}$ varied $\pm 4 \%$ due to changes in culture volume caused by variable stirrer speed; a dilution rate of $0 \cdot 1 \mathrm{~h}^{-1}$ varied $\pm 2 \%$.

Enzyme assays. Most of the enzyme assays employed have been described previously (Meiberg \& Harder, 1978).

Nitrate reductase was measured according to Boonstra et al. (1975).

Nitrite reductase was assayed by the method of Williams et al. (1978), except that 50 mM-potassium phosphate buffer was used and methylene blue was replaced by methylviologen. 
Dimethylamine mono-oxygenase in crude cell-free extracts was assayed according to Colby \& Zatman (1973). For assay of this enzyme activity in a partially purified dimethylamine mono-oxygenase preparation (used for kinetic and inhibition studies), the reaction mixture contained (in $1 \mathrm{ml}$ ) $100 \mu$ mol potassium phosphate buffer pH 7.0, variable amounts of $\mathrm{NADPH}$ and dimethylamine. $\mathrm{HCl}$, and enzyme. The reaction was started by adding dimethylamine.

All assays were performed in air-saturated solutions at $25^{\circ} \mathrm{C}$. For the determination of $K_{\mathrm{m}}$ for oxygen, the oxygen concentration in the assay mixtures was varied by bubbling air/nitrogen mixtures of different composition through the solution. The DOT in the reaction mixture was measured continuously with the vibrating $\mathrm{Ag} / \mathrm{Pt}$ oxygen-electrode accessory of an Aminco-Chance DW-2 spectrophotometer, which was used to record the rate of NADPH disappearance.

Partial purification of dimethylamine mono-oxygenase. Attempts to purify th edimethylamine monooxygenase from Hyphomicrobium $\mathbf{X}$ followed the procedure for the partial purification of the secondaryamine mono-oxygenase of Pseudomonas aminovorans (Brook \& Large, 1976). Hyphomicrobium X was grown in dimethylamine-limited continuous culture (dilution rate $0 \cdot 10 \mathrm{~h}^{-1}$ ) at a DOT of $75 \mathrm{mmHg}$. Effluent cell material was collected and stored at $-20^{\circ} \mathrm{C}$ until use. After three purification steps, including streptomycin sulphate treatment, ammonium sulphate fractionation (40 to $52 \%$ saturation) and Sephadex G-75 exclusion chromatography, all mono-oxygenase activity originally present in the crude cell-free extract was lost. In subsequent purifications, crude cell-free extracts were subjected to ammonium sulphate fractionation (40 to $60 \%$ saturation) only. The protein precipitate was dissolved in $50 \mathrm{~mm}$-potassium phosphate buffer pH 7.0 containing $5 \%(\mathrm{v} / \mathrm{v})$ ethanol; the latter was added to stabilize the preparation (Eady et al., 1971).

Oxygen solubility. The dissolved oxygen concentrations (in equilibrium with air at a $p \mathrm{O}_{2}$ of $0 \cdot 209$ atm) in the culture medium and assay mixtures were calculated to be $0.244 \mathrm{~mm}$ (at $25^{\circ} \mathrm{C}$ ) and $0.219 \mathrm{mM}$ (at $30^{\circ} \mathrm{C}$ ).

Chemicals. Methylammonium chloride, dimethylammonium chloride and trimethylammonium chloride were obtained from Merck. Methylviologen was from BDH. All other chemicals and reagents were from Sigma, Fluka or Merck. Biochemicals were from Boehringer.

\section{RESULTS}

Aerobic growth of Hyphomicrobium $X$ on trimethylamine, dimethylamine and methylamine

Recently Hyphomicrobium X was reported to grow at a specific rate of $0 \cdot 10 \mathrm{~h}^{-1}$ with trimethylamine as the substrate (Meiberg \& Harder, 1978). Accumulation and subsequent utilization of dimethylamine occurred during the second part of the exponential growth phase. This situation was similar to that described for anaerobic growth on trimethylamine in the presence of nitrate (Meiberg \& Harder, 1978). The dimethylamine which was produced from trimethylamine in the second growth phase accumulated in almost stoicheiometric amounts. Obviously it was not metabolized during this part of the growth curve. This was attributed to the inhibition of dimethylamine dehydrogenase by the trimethylamine present in the medium (Meiberg \& Harder, 1979). Dimethylamine dehydrogenase was the only dimethylamine-oxidizing enzyme detected under these conditions. The accumulation of dimethylamine during growth on trimethylamine was not observed in later experiments (Fig. 1). Trimethylamine was metabolized completely without the excretion of intermediary products such as dimethylamine and methylamine. In addition, the specific growth rate was now $0.13 \mathrm{~h}^{-1}$ as compared to $0 \cdot 10 \mathrm{~h}^{-1}$ in the previous experiments.

When Hyphomicrobium $\mathrm{X}$ was grown on dimethylamine as the growth substrate, accumulation and subsequent utilization of methylamine was observed (Meiberg \& Harder, 1978; Fig. 2). Whereas previously, growth on dimethylamine with a specific growth rate of $0 \cdot 12$ $\mathrm{h}^{-1}$ resulted only in a minor accumulation of methylamine, in the present experiments (Fig. 2), accumulation of this compound was more substantial (up to $4.6 \mathrm{~mm}$ ). After dimethylamine was depleted from the medium, growth occurred exclusively at the expense of methylamine (Fig. 2). As a result of this sequential substrate utilization the growth curve showed a biphasic pattern. During the first phase of growth, with dimethylamine as the substrate, the specific growth rate was high $\left(\mu=0.17 \mathrm{~h}^{-1}\right)$, whereas at the end of the growth curve, when growth occurred with the accumulated methylamine, the specific growth rate was lower $\left(0 \cdot 11 \mathrm{~h}^{-1}\right)$. This change in growth rate coincided with the depletion of dimethylamine from the medium and an increased rate of utilization of methylamine. Obviously 


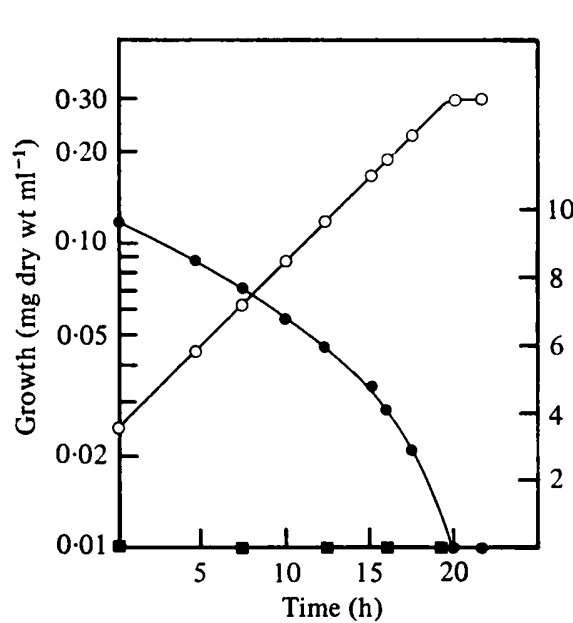

Fig. 1

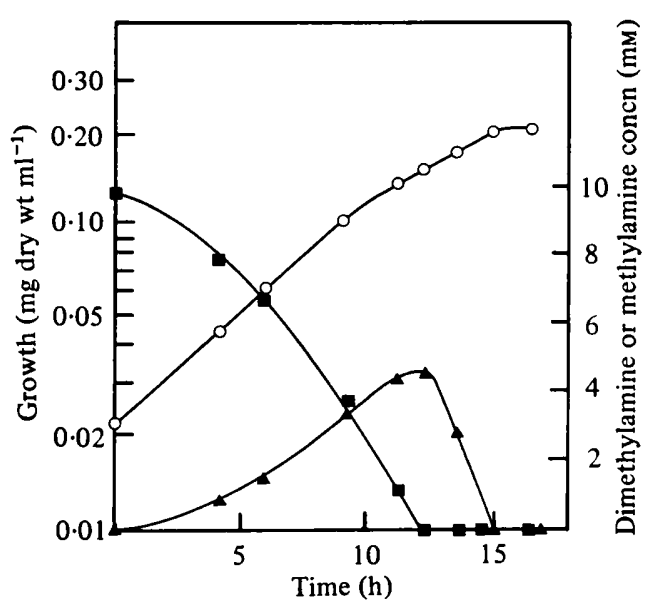

Fig. 2

Fig. 1. Aerobic growth of Hyphomicrobium $\mathrm{X}$ on trimethylamine: $\bigcirc$, growth; $\bigcirc$, trimethylamine and, $\square$, dimethylamine concentrations in the culture.

Fig. 2. Aerobic growth of Hyphomicrobium $X$ on dimethylamine: $\bigcirc$, growth; $\mathbf{D}$, dimethylamine and, $\boldsymbol{\Delta}$, methylamine concentrations in the culture.

\section{Table 1. Specific activities of various enzymes in crude extracts of Hyphomicrobium $X$} grown aerobically on trimethylamine and dimethylamine

Samples of Hyphomicrobium $\mathrm{X}$ grown on trimethylamine or dimethylamine were taken at the end of the active growth phase. Specific activities are expressed as nmol substrate transformed min $^{-1}$ (mg protein) $^{-1}$. Abbreviations: TMA, trimethylamine-grown organisms; DMA, dimethylaminegrown organisms; ND, not determined.

\section{Enzyme}

Trimethylamine dehydrogenase (PMS)

Trimethylamine mono-oxygenase (NADPH)

Trimethylamine- $N$-oxide aldolase

Dimethylamine dehydrogenase (PMS)

Dimethylamine mono-oxygenase (NADPH)
TMA DMA

$\begin{array}{rl}18(14)^{*} & 7(5) \\ 0(0) & 0(0) \\ 0(\mathrm{ND}) & 0(\mathrm{ND}) \\ 10(1) & 8(2) \\ 0(29) & 0(24)\end{array}$

* Data in parentheses refer to enzyme activities measured in crude extracts of cells grown in the experiments shown in Fig. 1 and 2. The other data are from Meiberg \& Harder (1978).

dimethylamine is a 'better' substrate for Hyphomicrobium $\mathrm{X}$ than methylamine. This is also evident from the maximum specific growth rate of $0.14 \mathrm{~h}^{-1}$ for growth of the organism on methylamine as the initial substrate under aerobic conditions (growth curves not shown).

Since growth of Hyphomicrobium $\mathrm{X}$ on methylamine as the initial substrate remained unaltered in the course of our studies, the different growth profiles of the organism on trimethylamine and dimethylamine must be ascribed to changes in the metabolism of trimethylamine to methylamine. The results of enzyme studies support this conclusion. Activities of trimethylamine- and dimethylamine-oxidizing enzymes were determined and compared with the results obtained previously (Meiberg \& Harder, 1978) (Table 1). Previously, dimethylamine dehydrogenase had been detected in cell-free extracts but in the present experiments (Fig. 1,2) the enzyme was barely detectable. Instead, dimethylamine mono-oxygenase, which could not be detected formerly, was now shown to be present in extracts of Hyphomicrobium $\mathrm{X}$ grown on trimethylamine and dimethylamine (Table 1). The presence of dimethylamine mono-oxygenase is most probably the basis for the now higher growth rate of the organism on trimethylamine and dimethylamine. The absence of accumu- 
Table 2. Comparison of some kinetic parameters of dimethylamine dehydrogenase and dimethylamine mono-oxygenase of Hyphomicrobium $X$

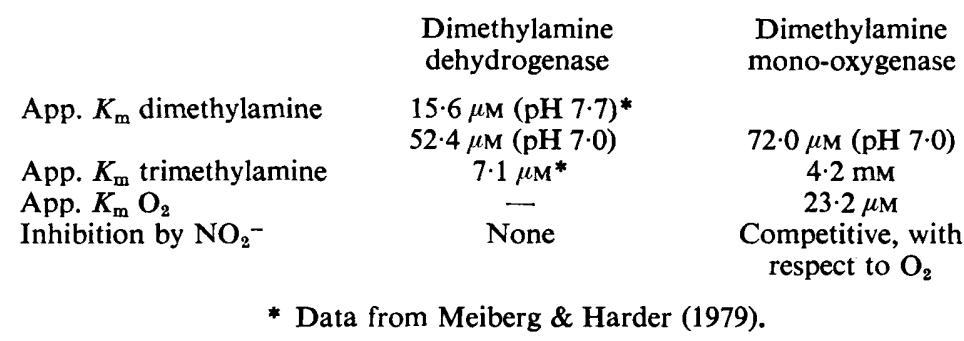

lation of dimethylamine during growth on trimethylamine, as shown in Fig. 1, may be explained by assuming that dimethylamine mono-oxygenase, unlike dimethylamine dehydrogenase, is not (or to a much lesser extent) inhibited by trimethylamine in the culture medium. Further details on this aspect are given below.

It seems likely that the different growth profiles and different growth rates of Hyphomicrobium $\mathrm{X}$ are related to differences in the oxidation of dimethylamine, because trimethylamine dehydrogenase activity had not changed significantly (Table 1). The conflicting results were neither caused by invalidity of the dimethylamine mono-oxygenase assay system used in previous experiments (as far as we were able to establish), nor by intrinsic alteration of the organism, nor by changes in medium composition. However, we have some inconclusive evidence for an inhibitory role of contaminating amines in the deionized water delivered to the laboratory up to 1977 . These compounds may have interfered with the activity or synthesis of dimethylamine mono-oxygenase in particular.

\section{Some properties of dimethylamine mono-oxygenase of Hyphomicrobium $X$}

In order to evaluate the effect of the DOT on the metabolism of methylated amines in Hyphomicrobium X, the kinetics of dimethylamine mono-oxygenase were studied. The properties of purified dimethylamine dehydrogenase have been described recently (Meiberg \& Harder, 1979). Special attention has been paid to a study of factors which may determine the relative contribution of dimethylamine mono-oxygenase and dimethylamine dehydrogenase to dimethylamine oxidation under in vivo conditions, namely the affinity of partially purified mono-oxygenase for its substrates (oxygen and dimethylamine) and its inhibition by trimethylamine and nitrite.

From a double-reciprocal plot of dimethylamine concentration against initial velocity of the reaction the apparent $K_{\mathrm{m}}$ of the mono-oxygenase for dimethylamine was calculated (Table 2). Similarly, the apparent $K_{\mathrm{m}}$ for NADPH was determined $(65.6 \mu \mathrm{M})$. These experiments were done at $0.244 \mathrm{~mm}-\mathrm{O}_{2}$ (air saturation at $25^{\circ} \mathrm{C}$ ). Under the same conditions the inhibition of dimethylamine mono-oxygenase by trimethylamine was investigated. Doublereciprocal plots of dimethylamine concentration against initial velocity at two different trimethylamine concentrations showed competitive inhibition of the mono-oxygenase by trimethylamine. An apparent $K_{1}$ value for trimethylamine was calculated from the slopes of the different plots (Table 2). Inhibition of the enzyme by trimethylamine was only very minor, compared with the effect of trimethylamine on dimethylamine dehydrogenase activity (Meiberg \& Harder, 1979; Table 2). At fixed non-saturating concentrations of NADPH $(0.15 \mathrm{~mm})$ and dimethylamine $(0.15 \mathrm{~mm})$ oxygen concentration was varied and the apparent $K_{\mathrm{m}}$ for molecular oxygen was calculated (Table 2). With $2 \mathrm{~mm}-\mathrm{NaNO}_{2}$ in the reaction mixture, competitive inhibition of dimethylamine mono-oxygenase by nitrite was observed with respect to oxygen. 


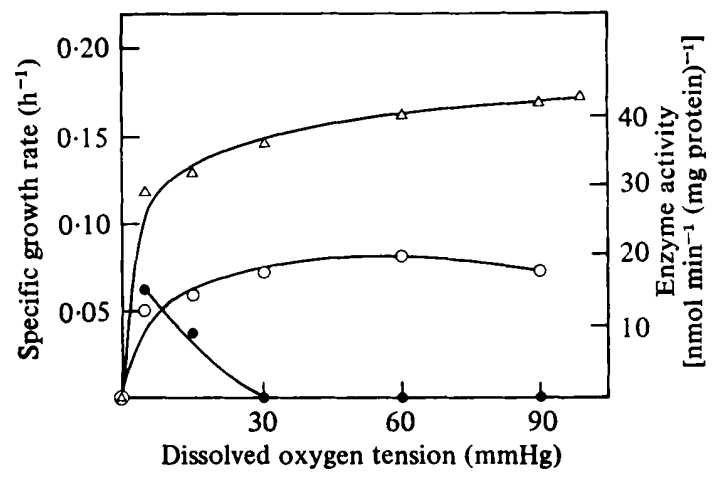

Fig. 3. Effect of DOT on the specific growth rate and enzyme activity of Hyphomicrobium $\mathrm{X}$ grown on dimethylamine in batch culture: $O$, dimethylamine mono-oxygenase activity; $O$, dimethylamine dehydrogenase activity; $\triangle$, specific growth rate.

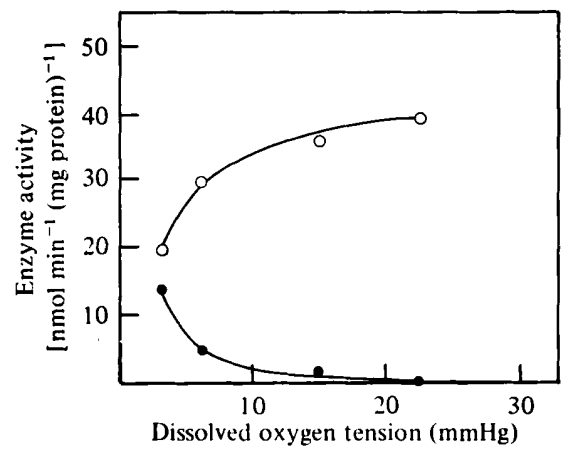

Fig. 4. Effect of DOT on the enzyme activity of Hyphomicrobium X grown on dimethylamine in continuous culture at a dilution rate of $0 \cdot 10 \mathrm{~h}^{-1}: 0$, dimethylamine mono-oxygenase activity; $\checkmark$, dimethylamine dehydrogenase activity.

\section{Role of the two dimethylamine-oxidizing enzymes at different oxygen concentrations in the presence and absence of nitrate}

Batch culture studies. When growth of Hyphomicrobium X on dimethylamine was followed in batch culture at different DOT values and cell-free extracts were assayed for dimethylamine mono-oxygenase and dehydrogenase activities, a dependence of growth rate and enzyme activity on DOT was observed (Fig. 3). The specific growth rate of the organism decreased only slightly with decreasing DOT down to about $15 \mathrm{mmHg}$; below this value it declined more rapidly from $\mu=0.13 \mathrm{~h}^{-1}$ to 0 . Dimethylamine mono-oxygenase activity followed the same pattern, whereas the dehydrogenase activity increased with decreasing DOT. It was not possible to determine whether the decreasing specific growth rates (as the results of decreasing DOT) determined the enzyme levels or whether the differences in enzyme synthesis, as a result of variation in DOT, caused the change in specific growth rate.

In order to study the influence of DOT on enzyme synthesis, experiments were carried out with chemostat cultures at a fixed dilution rate. Under these conditions any interference of a changing specific growth rate with enzyme synthesis was avoided.

Continuous culture studies-growth in the absence of nitrate. When Hyphomicrobium $\mathrm{X}$ was grown in continuous culture under dimethylamine limitation at a fixed dilution rate of $0 \cdot 10 \mathrm{~h}^{-1}$ and the DOT was varied from 30 to $3 \mathrm{mmHg}$, the dimethylamine mono-oxygenase activity in crude extracts of cells taken from a steady-state culture declined with decreasing DOT, whereas dimethylamine dehydrogenase activity increased (Fig. 4). This 


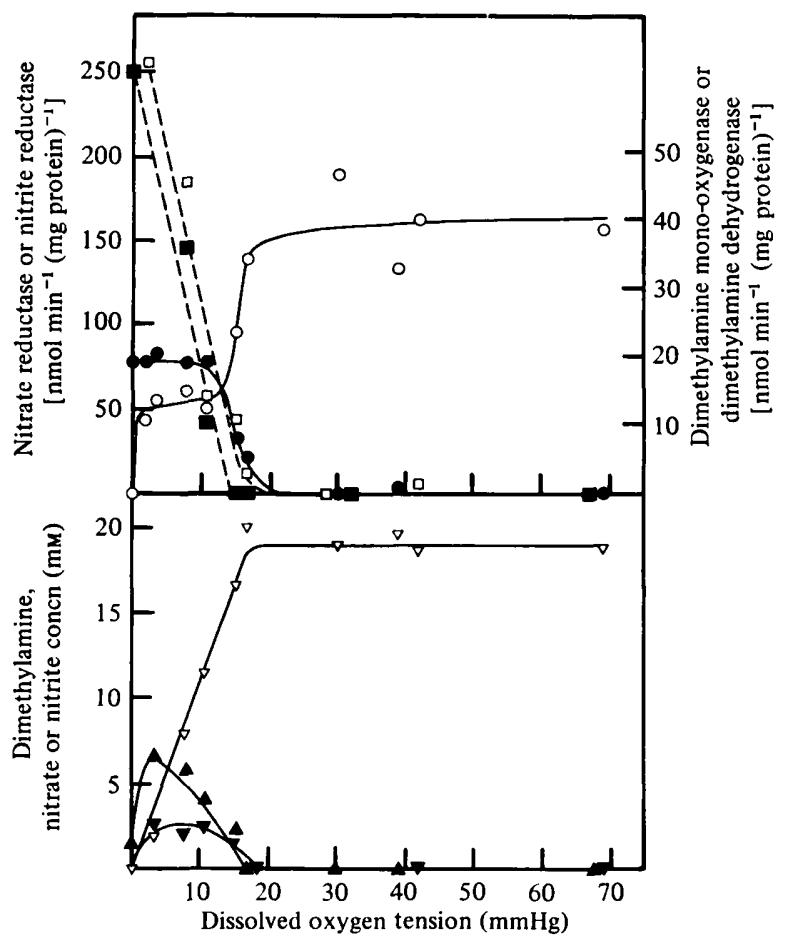

Fig. 5. Effect of DOT on the enzyme activity of Hyphomicrobium X grown on dimethylamine in continuous culture at a dilution rate of $0 \cdot 10 \mathrm{~h}^{-1}$ with nitrate in the growth medium: $\bigcirc$, dimethylamine mono-oxygenase activity; $\bigcirc$, dimethylamine dehydrogenase activity; $\square$, nitrate reductase activity; $\boldsymbol{\nabla}$, nitrite reductase activity; $\boldsymbol{\Delta}$ dimethylamine, $\nabla$, nitrate and, $\boldsymbol{\nabla}$, nitrite concentrations in the culture fluid.

pattern is not essentially different from that observed in batch culture. It seems likely that at lower DOT values the dehydrogenase becomes increasingly important and the monooxygenase less important. In view of the fact that oxygen is indirectly required for the oxidation of dimethylamine by dimethylamine dehydrogenase (namely, for the oxidation of the reduced cofactor of the enzyme), it is not surprising that at a DOT of $3 \mathrm{mmHg}$ or below, oxygen becomes growth-rate limiting. At a DOT of $3 \mathrm{mmHg}$, dimethylamine was detected in the culture fluid, whereas at higher DOT the substrate concentration remaining in the culture was undetectably low. At a DOT lower than $3 \mathrm{mmHg}$, the culture washed out as the growth rate became limited by oxygen availability and decreased to below a value corresponding to the dilution rate of $0 \cdot 10 \mathrm{~h}^{-1}$.

As such, these experiments did not yield information on the actual enzyme activity at, or just above, zero DOT. However, this range of oxygen tensions may be very important for hyphomicrobia which have the potential to denitrify. Therefore, in the following experiments nitrate was also supplied to the culture.

Continuous culture studies - growth in the presence of nitrate. Growth of Hyphomicrobium $\mathrm{X}$ on dimethylamine in continuous culture at different DOT values at a dilution rate of $0 \cdot 10 \mathrm{~h}^{-1}$ in the presence of $20 \mathrm{~mm}$-nitrate did not result in wash-out of the culture at any of the DOT values tested - from 0 to about $70 \mathrm{mmHg}$. This indicated that, especially at zero or very low oxygen tensions where the main electron acceptor must be nitrate, the growth rate was sufficiently high to maintain a steady state at a dilution rate of $0 \cdot 10 \mathrm{~h}^{-1}$ under these denitrifying conditions, although in batch culture growth under anaerobic conditions was very slow (Meiberg \& Harder, 1978). 


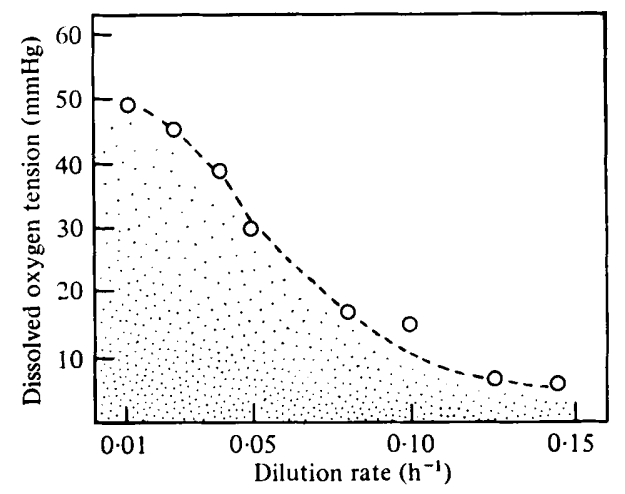

Fig. 6. Correlation between dilution rate and DOT with respect to induction of detectable activity of nitrate reductase in Hyphomicrobium $\mathrm{X}$ grown on dimethylamine in continuous culture in the presence of nitrate: $\bigcirc$ minimal, but significant, nitrate reductase activity [about $10 \mathrm{nmol}$ nitrate transformed $\min ^{-1}$ (mg protein $\left.)^{-1}\right]$. The dotted area represents conditions where denitrification occurs. In this area nitrate reductase activity most probably increased rapidly with decreasing DOT.

Considering the change in specific activities of dimethylamine mono-oxygenase and dimethylamine dehydrogenase with DOT, the general tendency observed in the previous experiments (Fig. 3, 4) was again recognized (Fig. 5), although there were some minor differences in the profiles of specific enzyme activities. Dimethylamine mono-oxygenase activity decreased suddenly at a DOT of $15 \mathrm{mmHg}$ to one-third of its original activity and remained constant at this low level when the DOT was further decreased to zero. At undetectably low DOT, dimethylamine mono-oxygenase remained present as long as oxygen was provided to the culture; when oxygen was excluded and complete anaerobiosis was introduced, mono-oxygenase activity declined to zero. In contrast, dimethylamine dehydrogenase activity which was not detected at DOT values above $30 \mathrm{mmHg}$, increased rapidly at a DOT of $15 \mathrm{mmHg}$ and reached a plateau at $10.5 \mathrm{mmHg}$ and below. It is notable that at the same DOT value $(15 \mathrm{mmHg})$ where enzyme activities changed dramatically, induction of nitrate reductase and nitrite reductase was observed. Both enzymes showed a linear increase in activity with decreasing DOT. At the same time, nitrate consumption and nitrite accumulation was detected (Fig. 5). At DOT below $10 \mathrm{mmHg}$, dimethylamine monooxygenase is probably inactive as a result of the low oxygen tension and the relatively 'high' nitrite concentration (Table 2). This suggests that dimethylamine dehydrogenase must be active in this range of DOT although possibly not optimally in view of the accumulation of dimethylamine. It is also possible that nitrite interferes with the dissimilation of dimethylamine, for the appearance of nitrite in the culture coincided with that of dimethylamine. Nitrite and dimethylamine concentrations decreased simultaneously when the DOT approached zero. Under strictly anaerobic conditions both nitrate and nitrite were exhausted from the culture medium while some dimethylamine $(1.35 \mathrm{~mm})$ was left.

Identical experiments have been performed at a lower dilution rate $\left(D=0.05 \mathrm{~h}^{-1}\right)$. Under these conditions similar phenomena were observed with one major difference: the DOT at which dimethylamine mono-oxygenase activity decreased, and dimethylamine dehydrogenase increased with the simultaneous occurrence of denitrification, was shifted towards higher values (about $45 \mathrm{mmHg}$ ). These results suggested that, besides the presence of nitrate, oxygen tension is not the only factor responsible for induction of denitrification growth rate may also play a role. 


\section{Effect of specific growth rate on the onset of nitrate reduction at different dissolved oxygen tensions}

From the experiments described above it is tempting to speculate that nitrate reduction in Hyphomicrobium $\mathrm{X}$ is switched on at higher DOT as the growth rate of the culture is lower. This suggestion was borne out by further continuous culture experiments in which at several fixed dilution rates DOT was decreased until nitrate reductase activity became apparent (Fig. 6). In view of the linear increase in nitrate reductase activity with decreasing DOT at dilution rates of $0.10 \mathrm{~h}^{-1}$ (Fig. 5) and $0.05 \mathrm{~h}^{-1}$ (results not shown), it is reasonable to assume a similar relationship at other dilution rates. This indicates that once nitrate reduction becomes apparent, its activity will increase rapidly as the DOT is further decreased. Thus, at low dilution rates, denitrification occurs over a wide range of DOT ( 0 to $50 \mathrm{mmHg}$ at $D=0.01 \mathrm{~h}^{-1}$ ). However, at dilution rates close to $\mu_{\max }$ the DOT range at which denitrification occurs is very small and approaches zero. Hyphomicrobium X may not be different from many other micro-organisms which have been reported to denitrify only under anaerobic conditions or at very low DOT. It may be that also in these microorganisms, when studied more closely, denitrification is induced at relatively high DOT at (very) low growth rates.

\section{DISCUSSION}

Hyphomicrobium $\mathrm{X}$ possesses two different enzymes for the oxidation of dimethylamine, namely dimethylamine dehydrogenase and dimethylamine mono-oxygenase. Anaerobic growth is only possible with dimethylamine dehydrogenase because the mono-oxygenase is inactive in the absence of oxygen. Although Hyphomicrobium X was able to grow aerobically with dimethylamine dehydrogenase as the functional enzyme (Meiberg \& Harder, 1978), the mono-oxygenase enables the organism to grow at a faster rate (Fig. 2). Some factors that characterize the properties of both enzymes are presented in Table 2 . The apparent $K_{\mathrm{m}}$ for dimethylamine of both enzymes when determined at $\mathrm{pH} 7.0$ is of the same order of magnitude. Although the maximum specific activities of the two enzymes, when measured in crude extracts, were comparable, very little can be said about the in vivo enzyme activity because both enzymes have completely different electron-transferring mechanisms. Nevertheless, the observation that Hyphomicrobium $\mathrm{X}$ is able to grow faster when dimethylamine mono-oxygenase is present suggests that in vivo this enzyme is capable of a higher rate of oxidation of dimethylamine than is dimethylamine dehydrogenase.

Trimethylamine is a potent inhibitor of dimethylamine dehydrogenase activity (Meiberg \& Harder, 1979). In contrast, dimethylamine mono-oxygenase is only slightly inhibited by relatively high trimethylamine concentrations. This different affinity for trimethylamine may explain the absence of accumulating dimethylamine in the culture medium during growth on trimethylamine when Hyphomicrobium X contained dimethylamine mono-oxygenase as the only functional enzyme (Fig. 1), whereas dimethylamine accumulated during growth of the organism on trimethylamine when only dimethylamine dehydrogenase was present (Meiberg \& Harder, 1978).

Another main difference between the two enzymes is their relation towards oxygen. The activity of dimethylamine dehydrogenase is independent of oxygen. Dimethylamine monooxygenase is strictly dependent on oxygen since this is a substrate for the enzyme. The apparent $K_{\mathrm{m}}$ for oxygen $(23.2 \mu \mathrm{M})$ of dimethylamine mono-oxygenase indicates that at a DOT of about $15 \mathrm{mmHg}$ the enzyme has an activity which is only half $V_{\max }$. Thus, at low DOT the mono-oxygenase is less active; this emphasizes the importance of dimethylamine dehydrogenase under these conditions, particularly when the growth medium contains nitrate which can replace oxygen as terminal electron acceptor.

In Hyphomicrobium $\mathrm{X}$ not only the activity but also the synthesis of both dimethylamine mono-oxygenase and dimethylamine dehydrogenase is regulated in response to variations 
in oxygen tension. The present results clearly show that higher oxygen tensions repress the synthesis of dimethylamine dehydrogenase. Repression is released when DOT is decreased. Dimethylamine mono-oxygenase is absent when no oxygen is supplied to cultures of the organism, but induction takes place as soon as oxygen is available. However, oxygen is not the only factor that regulates the synthesis of the two enzymes. Both the mono-oxygenase and the dehydrogenase, being catabolic enzymes, are also induced by their substrate dimethylamine, because in methylamine-grown cells these enzymes are undetectable (Meiberg \& Harder, 1978).

The addition of nitrate to the growth medium does not alter the observed enzyme patterns essentially. In fact, the process of denitrification in Hyphomicrobium $\mathrm{X}$ does not require major changes in the main metabolic features of the organism, because anaerobically grown cells are still able to respire with oxygen (Large et al., 1979). Denitrification only requires the additional synthesis of the enzymes which reduce nitrate to $\mathrm{N}_{2}$, as well as some quantitative shifts in the components of the electron transport chain (see Large et al., 1979). The only factor which may have a major impact on the metabolism of methylated amines is nitrite, which accumulated during growth of Hyphomicrobium $\mathrm{X}$ in continuous culture under denitrifying conditions (Fig. 5). Nitrite has been frequently found to accumulate in batch cultures (Payne, 1973) as a transient intermediate in denitrification of nitrate to $\mathrm{N}_{2}$. During anaerobic growth of Hyphomicrobium X in batch culture we also observed accumulation of nitrite (Meiberg \& Harder, 1978). Nitrite accumulation may be explained by inhibition of nitrite reductase by high nitrate concentrations. Another possibility is that nitrite reductase activity is too low to reduce all the nitrite formed by the action of nitrate reductase.

At present, it is not precisely known where nitrite exerts its inhibition. Rowe et al. $(1977,1979)$ suggested that nitrite interferes with the flow of electrons along the electron transport chain. They found in Pseudomonas aeruginosa that nitrite inhibited active transport, oxygen uptake and oxidative phosphorylation. It was concluded that nitrite oxidized the $\mathrm{Fe}^{2+}$ of electron carriers, such as in the terminal cytochrome oxidase, to $\mathrm{Fe}^{3+}$. Recently Meijer et al. (1979) demonstrated in Paracoccus denitrificans dissipation of the protonmotive force by nitrite. Nitrite renders the cell membrane permeable to protons, causing a destruction of the $\Delta \mathrm{pH}$ component. In order to explain the accumulation of dimethylamine during growth of Hyphomicrobium $\mathrm{X}$ in continuous culture with dimethylamine as the growth substrate (Fig. 5), we must assume that nitrite limits the rate of energy generation from dimethylamine oxidation in the way suggested by Rowe et al. $(1977,1979)$, rather than by uncoupling. It is noteworthy that the appearance of nitrite in the culture coincided with that of dimethylamine (Fig. 5). Oxygen-uptake experiments with whole cells of Hyphomicrobium $\mathrm{X}$ (with methanol as the substrate) showed strong inhibition of oxidative respiration (e.g. cytochrome oxidase activity) by nitrite at relatively low DOT values. This may suggest that, although oxygen is present during continuous cultivation, oxidative respiration is impossible in the presence of nitrite (Fig. 5).

The process of denitrification in Hyphomicrobium $\mathrm{X}$ is inducible, as in all other denitrifying micro-organisms (Payne, 1973). Not only oxygen tension is important but also the presence of nitrate (or nitrite). Nitrate reductase could not be detected in cell-free extracts of Hyphomicrobium $\mathrm{X}$ grown in continuous culture on dimethylamine in the absence of nitrate. In addition, specific growth rate appeared to affect induction of nitrate reductase (Fig. 6). During growth on dimethylamine at low dilution rates nitrate reductase was present at higher DOT values than during growth at higher dilution rates. The consumption of nitrate and the accumulation of nitrite indicated the occurrence of denitrification at relatively high oxygen tensions. The same phenomenon was reported recently for Klebsiella sp. strain $\mathrm{K} 312$, when grown in continuous culture at a dilution rate of $0.10 \mathrm{~h}^{-1}$ (Dunn et al., 1979). At present, it is not known whether the denitrification potential of this organism is growth rate-dependent. 
In addition to the oligocarbophilic nature of hyphomicrobia (e.g. Harder \& Attwood, 1978), the capacity for 'aerobic' denitrification at relatively high oxygen tensions at low specific growth rates reported in this paper may be of ecological importance.

\section{REFERENCES}

Boonstra, J., Huttunen, M. T., Konings, W. N. \& KABACK, H. R. (1975). Anaerobic transport in Escherichia coli membrane vesicles. Journal of Biological Chemistry 250, 6792-6798.

Brook, D. F. \& Large, P. J. (1976). A steady-state kinetic study of the reaction catalysed by the secondary-amine mono-oxygenase of Pseudomonas aminovorans. Biochemical Journal 157, 197-205.

Colby, J. \& Zatman, L. J. (1973). Trimethylamine metabolism in obligate and facultative methylotrophs. Biochemical Journal 132, 101-112.

Colby, J. \& Zatman, L. J. (1975). Enzymological aspects of the pathways for trimethylamine oxidation and $\mathrm{C}_{1}$ assimilation in obligate methylotrophs and restricted facultative methylotrophs. Biochemical Journal 148, 513-520.

Dunn, G. M., Herbert, R. A. \& Brown, C. M. (1979). Influence of oxygen tension on nitrate reduction by a Klebsiella sp. growing in a chemostat culture. Journal of General Microbiology 112, 379-383.

EADY, R. R., Jarman, T. R. \& LARGe, P. J. (1971). Microbial oxidation of amines. Partial purification of a mixed-function secondary-amine oxidase system from Pseudomonas aminovorans that contains an enzymically active cytochromeP-420-type haemoprotein. Biochemical Journal 125, 449-459.

Hampton, D. \& Zatman, L. J. (1973). The metabolism of tetramethylammonium chloride by Bacterium 5H2. Biochemical Society Transactions 1, 667-668.

Harder, W. \& Attwood, M. M. (1978). Biology, physiology and biochemistry of hyphomicrobia. Advances in Microbial Physiology 17, 303-359.

HARder, W., Visser, K. \& Kuenen, J. G. (1974). Laboratory fermenter with an improved magnetic drive. Laboratory Practice 23, 644-645.

HiRSCH, P. \& ConTI, S. F. (1964). Biology of budding bacteria. II. Growth and nutrition of Hyphomicrobium spp. Archiv für Mikrobiologie 48, 358-367.

Large, P. J., Meiberg, J. B. M. \& Harder, W. (1979). Cytochrome $c_{\mathrm{CO}}$ is not a primary electron acceptor for the amine dehydrogenases of Hypho- microbium X. FEMS Microbiology Letters 5, 281-286.

Loginova, N. V. \& Trotsenko, Yu. A. (1976). Pathways of oxidation and assimilation of methylated amines in Arthrobacter globiformis. Microbiology (English translation of Mikrobiologiya) 45, 196-200.

Loginova, N. V., Shishkina, V. N. \& Trotsenko, Yu. A. (1976). Primary metabolic pathways of methylated amines in Hyphomicrobium vulgare. Microbiology (English translation of Mikrobiologiya) 45, $34-40$.

Meiberg, J. B. M. \& Harder, W. (1978). Aerobic and anaerobic metabolism of trimethylamine, dimethylamine and methylamine in Hyphomicrobium X. Journal of General Microbiology 106, 265-276.

Meiberg, J. B. M. \& Harder, W. (1979). Dimethylamine dehydrogenase from Hyphomicrobitum X: purification and some properties of a new enzyme that oxidizes secondary amines. Journal of General Microbiology 115, 49-58.

MeiJer, E. M., van DeR ZwaAn, J. W., WeVer, R. \& Stouthamer, A. H. (1979). Anaerobic respiration and energy conservation in Paracoccus denitrificans. Functioning of iron-sulfur centers and the uncoupling effect of nitrite. European Journal of Biochemistry 96, 69-76.

PAYNE, W. J. (1973). Reduction of nitrogenous oxides by microorganisms. Bacteriological Reviews 37, 409-452.

Rowe, J J., Hodge, III, T. W. \& EAGoN, R. G. (1977). Nitrite inhibition of active transport and of respiration in Pseudomonas aeruginosa K226. Abstracts of the Annual Meeting of the American Society for Microbiology, p. 223.

Rowe, J. J., Yarbrough, J. M., RAKe, J. B. \& EAGON, R. G. (1979). Nitrite inhibition of aerobic bacteria. Current Microbiology 2, 51-54.

VishniaC, W. \& SANTER, M. (1957). The thiobacilli. Bacteriological Reviews 21, 195-213.

Williams, D. R., Rowe, J. J., Romero, P. \& EAGoN, R. G. (1978). Denitrifying Pseudomonas aeruginosa: some parameters of growth and active transport. Applied and Environmental Microbiology 36, 257-263. 